\title{
EFFECT OF COMPOST AND SEAWEED EXTRACT ON THE PHYSIOLOGICAL CHARACTERISTICS OF MARKETABLE FRUITS OF TWO SWEET PEPPER (Capsicum annuUm L.) HYBRIDS
}

\author{
NASHWAN Y. EIDO and DR. SARFARZ F. A. AL-BAMARNY \\ Dept. of Horticulture, College of Agricultural Engineering Sciences, University of Duhok, \\ Kurdistan Region-Iraq
}

(Received: August 5, 2019; Accepted for Publication: September 29, 2019)

\begin{abstract}
The experiment was conducted on two hybrids (Massouda and Chahin) sweet pepper (Capsicum annuum L.) in the field of the College of Agricultural Engineering Sciences, University of Duhok during growing season of 2018, to study the effects of three levels of artificial compost in soil $(0: 0,1: 1,1: 3 \mathrm{v} / \mathrm{v})$ and four concentrations of seaweed extract spray (Tecamin algae) $\left(0.0,0.5,0.75,1 \mathrm{ml}^{.} \mathrm{.}^{-1}\right)$ on Massouda F1 and Chahin F1 hybrids. The results shows that Massouda hybrid significantly recorded higher fruit dry weight and vitamin $\mathrm{C}$, whereas Chahin hybrid were overcome significantly from Massouda in chlorophyll $a$ and $b$. The high level 1:1 compost significantly increased fruit dry weight, vitamin $C$ and total carbohydrate, while the level 1:3 compost significantly recorded higher chlorophyll a and lower fruit respiration rate compared to untreated hybrids with compost. However, the influence of high concentration $\left(1 \mathrm{mll}^{-1}\right)$ seaweed on the marketability pepper fruits was affected significantly and improved fruit dry weight, vitamin $C$, total carbohydrate, chlorophyll $b$ and lower fruit respiration rate than untreated with seaweed except chlorophyll a which significantly increased in level $\left(0.5 \mathrm{mll}^{\mathrm{I}^{-1}}\right)$ seaweed. On the other hand, the triple interactions among the three factors significantly enhanced all detected traits compared to untreated plants.
\end{abstract}

KEYWORDS: Sweet pepper, (Capsicum annuum L.), artificial compost, seaweed algae, fruit marketable.

https://doi.org/10.26682/ajuod.2019.22.2.15

\section{INTRODUCTION}

C apsicum (Capsicum annuum L.) commonly known as bell pepper or sweet pepper or green pepper belongs to the family Solanaceae along with tomatoes, eggplant and potatoes (Safford, 1926), and is native to the Central and South America (Mexico) (Cheng, 2014). It consider as a popular vegetable crop produced worldwide. Consumption of sweet peppers is becoming popular due to their medicinal and varied flavours, aroma, colour and health promoting compounds (vitamin C, vitamin $\mathrm{E}$ and carotenoids) (Raffo et al., 2007).

Capsicum is the most widely summer vegetable crops cultivated and economically planted in many countries all over the world includes sweet and pungent fruit of numerous shapes and sizes (Rubatzky, 1997). It is the world's second most important vegetable after tomato (Anonymous, 1989).
Pepper is one of the most valuable vegetable crops in Iraq and very desirable for Iraqi consumer. According to the United States Agency for International Development (USAID) in (2011-2012) the production yield of bell peppers in Iraq ranged from 8 to 14 tons/donums.

Nowadays farmers are interested in moving to organic cultivation. However, organic farming is one of the fastest growing sectors of agriculture worldwide (Karanatsidis and Berova, 2009). Fertilizers and soil amendments are a wide array of materials added to soils to improve plant growth. It is more than three decades that the global agriculture has been depicted towards organic agriculture owing to sustainability and reduced environmental effects (Adhikari et al., 2016). The use of compost and seaweed extract has assumed a great significance in recent years in vegetables production. However, in many investigations, compost utilized as a soil amendment has resulted in higher marketable yields and fruit quality as 
compared with those from standard vegetable crop commercial practices (Stoffella et al., 2003). Compost not only supplies many nutrients for crop production, but they are also valuable sources of organic matter. The use of composts has been used to increase crop productivity and yields, and their use is usually associated with improved soil structure and enhanced soil fertility, increased soil microbial populations and activity and an improved moisture-holding capacity of the soil (Arancon et al., 2004).

The use of other organic fertilizers such as seaweed extract as a liquid manure in farming practice is very ancient and common practice among the Romans and also practiced in Britain, France, Spain, Japan and China (Arumugam and Anantharaman, 2009). Seaweeds have been applied as foliar spray and used in organic farming. These extracts are marketed as liquid fertilizers and bio-stimulants, because they contain growth promoting substances such as Auxins and cytokinins that induce the shoot and root system consistently (Stirk et al., 2004).

Zaki et al., (2017) showed that among five pepper cultivars, the hot red and sweet red pepper had high vitamin $\mathrm{C}$ content, followed by hot green pepper, sweet green pepper and yellow pepper. Hallmann and Rembialkowska (2012) showed that organic bell pepper fruits contained significantly more fruit dry matter, vitamin $\mathrm{C}$ compared with conventional fruits. Aminifard et al., (2013) studied the effect of compost on fruit quality of sweet pepper, the results reported that pepper fruit carbohydrate increased with increasing compost levels and the lowest carbohydrate was recorded in control, Also showed that vitamin $\mathrm{C}$ in fruit quality influenced by compost treatments. The results of Manna $\boldsymbol{e t}$ al., (2012) showed that seaweed extract led to increasing yield, chlorophylls and vitamin $\mathrm{C}$ in chilli pepper compared to those without seaweed. However, Vijayakumar et al., (2018) showed that application of seaweed on sweet pepper fruit recorded maximum fruit dry weight, carbohydrate content, chlorophyll a, b and total chlorophyll.

Therefore the present study objectives were to determine the effect of compost as a soil amendment with seaweed extract as a foliar spray and their interactions on the physiological characteristics of peppers and improving marketable fruits of organic sweet pepper plants.

\section{MATERIALS AND METHODS}

The experiment was conducted under field conditions on $5^{\text {th }}$ March (2018) at the research farm of the College of Agricultural Engineering Sciences, University of Duhok, Kurdistan region-Iraq. The site is located at Sumail near $13 \mathrm{~km}$ west of Duhok city at (Latitude $36.51 \mathrm{~N}$, Longitude $42.52 \mathrm{E}$ and $473 \mathrm{~m}$ altitude). Soil sampling, preparation and analysis were analysed (data not presented).

Two hybrids of sweet pepper (Capsicum annuum L.), Massouda RZ F1 and Chahin F1 (purity $99.9 \%$ - 98\% and germination 97\% 93\%) respectively were purchased from a local distributor. During 5/3/2018 Pepper seeds $(C$. annuum L.) sowing in plastic pots $(6 \mathrm{~cm}$ diameter, one seed per pot) each pots containing $50 \mathrm{~g}$ of peat moss. After sowing, the pots were placed in a greenhouse and after 44 days the seedling transported into the field.

Aerobically composted grass clippings with other organic matter were done. Grass clippings were obtained from local gardens (Table 1). 
Table (1): Compost ingredients.

\begin{tabular}{|c|c|c|}
\hline Materials & Amount & Reference \\
\hline Grass clippings & $100 \mathrm{~kg}$ & Optional \\
\hline Poultry manure & $40 \mathrm{~kg}$ & Adediran et al., 2003 \\
\hline Balk agents (Millet) & $20 \mathrm{~kg}$ & Epstein, 2011 \\
\hline Clay soil & $10 \mathrm{~kg}$ & CASFS $^{1}, 2017$ \\
\hline Molasses & $3 \mathrm{~kg}$ & Duffy et al., 2013 \\
\hline Calcium carbonate & $3 \mathrm{~kg}$ & Baysal et al., 2007 \\
\hline Bio-char (Charcoal) & $5 \%$ & Khan et al., 2014 \\
\hline $\mathrm{pH}$ & 7.6 & Rittenhouse, 2015 \\
\hline (Water) Moisture & $50 \%$ & \\
\hline Temperature & $59^{\circ} \mathrm{C}$ & \\
\hline Particle size & $\sim 2.5 \mathrm{~cm}$ & Varies $^{2}$ \\
\hline ass clippings $(\mathrm{C} / \mathrm{N})$ ratio & $15-25: 1$ & $\begin{array}{c}\text { Compost management program (A and L Canada } \\
\text { Laboratories, (2004) }\end{array}$ \\
\hline
\end{tabular}

1. (CASFS): A Project for the Center of Agroecology and Sustainable Food Systems. University of California, Santa Cruz. Unit 1.7. Making and Using. Compost, (casfs.ucsc.edu).

2. Depends on the specific materials and pile size.

Compost factor consisted of three levels, involving, compost + soil $(0: 1,1: 1$ and $1: 3 \mathrm{v} / \mathrm{v})$ ratios (Llaven et al., 2008). Compost was applied to the experimental plots at the time with transplanting the pepper seedlings in to the field in (20/4/2018) and the plots media was prepared by mixing soil collect from upland orchard and mix with the amount of compost as levels per the experimental units. Compost application to the experimental plots was be mixed with soil in hole $(20 \mathrm{~cm}$ diameter and $30 \mathrm{~cm}$ depth), the hole filled with the compost and soil before pepper plantlet transplantation and watering was done through drip irrigation system.

The seaweed extract (Tecamin algae, made in spain, Agritecno Fertilizantes Company) obtained from local distributor. contains; seaweed extract $16 \%(\mathrm{w} / \mathrm{w})$, total Nitrogen $0.1 \%$, Phosphorus $\left(\mathrm{P}_{2} \mathrm{O}_{5}\right) \quad 0.15 \%$, Potassium $\left(\mathrm{K}_{2} \mathrm{O}\right) 0.25 \%(\mathrm{w} / \mathrm{w})$, trace elements\%, growth regulator $\mathrm{L}^{-1}$ (bio-stimulants), vitamin $\%$, amino acid\% and carbohydrates\%. Four different concentration of seaweed extract $(0.0,0.5,0.75$ and $\left.1 \mathrm{ml.} \mathrm{l}^{-1}\right)$ was used after a week of transplanting and spray of seaweed repeat every 10 days during vegetative growing season until fruit harvesting. The total spray of seaweed extract was done at six times.
The experiment was arranged in a split-split plot design in RCBD, there were three factors under the study, two hybrids of sweet pepper, three levels of compost and four concentrations of seaweed extract $(2 \times 3 \times 4)$ with three replications and twenty four treatments. In splitsplit plot design there are three types of experimental units (main plots, sub plots and sub-sub plots). Where the levels of hybrids were putted in a main plots, and three levels of compost were putted in the sub-plots, while four concentrations of seaweed extract was putted in the sub-sub plots. Data was analysed using SAS program and means was compared by Duncan's multiple range test at $5 \%$ level of confidence.

\section{Measurements}

Fruit marketable quality after 10 days at $\mathbf{2 2}$ ${ }^{\circ} \mathbf{C}$ : At the middle of growing season more than 25 fruits from each treatment were kept at temperature $22{ }^{\circ} \mathrm{C}$ for 10 days, then the physiological changes that occurred in the fruits quality of sweet pepper within ten days at $22{ }^{\circ} \mathrm{C}$ was determined at the following methods:

Fruit dry weight (\%): Cut $120 \mathrm{~g}$ of fruits under each experimental unit was taken for fruit dry weight according to (Gobara, 1998).

Vitamin C (ascorbic acid) (\%): Pepper juice extracted from five fruits then ascorbic acid was 
determined using 2, 6 Dichloro-phenol indophenols as described in (A.O.A.C, 2000).

Fruit respiration rate $\left(\mathrm{mg} \mathrm{CO}_{2} / \mathrm{kg}\right.$-h): Fruit respiration was measured using closed system (desiccator) method according to (Al-any, 1985) that calculates the amount of $\mathrm{CO}_{2}$ liberated from the fruits and absorbed by sodium hydroxide.

$$
2 \mathrm{NaOH}+\mathrm{CO}_{2} \rightarrow \mathrm{Na}_{2} \mathrm{CO}_{3}+\mathrm{H}_{2} \mathrm{O}
$$

Carbohydrate contents (\%): Total carbohydrates were determined according to Lane and Eynon method as described by Joslyn in (1970).

Fruit chlorophyll content (a and b) (mg.g $\left.{ }^{-1}\right)$ : For determining chlorophyll $\mathrm{a}$ and $\mathrm{b}$ of fruit was calculated according to Knudsen method as described by Wintermans and DeMots (1965).

\section{RESULTS \\ Fruit dry weight (\%)}

The Percentage of fruit dry matter shows significantly increase $(10.36 \%)$ in Massouda hybrid compared to $(8.09 \%)$ in Chahin. The highest rate $(9.68 \%)$ of fruit dry weight recorded in a high level 1:1 compost which was significantly higher compared with untreated plant. The effect of seaweed concentration at 1 $\mathrm{ml} . \mathrm{l}^{-1}$ were also increased significantly $(10.04 \%)$ fruit dry matter compared to control which recorded the lowest rate $(9.04 \%)$ and other seaweed concentrations. The interactions among hybrids, compost and seaweed were increased the percentage of fruit dry weight as shown in Table (2) whereas Chahin planted in high level of compost 1:1 and sprayed with high level of seaweed $1 \mathrm{ml}^{-1} \mathrm{l}^{-1}$ increased significantly fruit dry weight to $(12.42 \%)$ compared to the most other interactions, the lowest (7.36\%) fruit dry weight measured in Chahin with 0:1 compost and 0.1 seaweed.

Table (2): Effect of compost, seaweed and their interactions on fruit dry weight (\%) of two marketable sweet pepper hybrids after 10 days at temperature $22^{\circ} \mathrm{C}$.

\begin{tabular}{|c|c|c|c|c|c|c|c|}
\hline \multirow[t]{2}{*}{ Hybrids } & \multirow{2}{*}{$\begin{array}{l}\text { Compost + } \\
\text { Soil (v/v) }\end{array}$} & \multicolumn{4}{|c|}{ Seaweed levels ml. $\mathrm{L}^{-1}$} & \multirow{2}{*}{$\begin{array}{l}\text { Hybrids } x \\
\text { Compost }\end{array}$} & \multirow{2}{*}{$\begin{array}{c}\text { Means } \\
\text { of } \\
\text { hybrids }\end{array}$} \\
\hline & & 0 & 0.5 & 0.75 & 1 & & \\
\hline \multirow[t]{3}{*}{ Massouda } & 0.1 & $10.00 \mathrm{~cd}$ & $10.57 \mathrm{bc}$ & $10.06 \mathrm{~cd}$ & $10.85 \mathrm{bc}$ & $10.37 b$ & $10.75 \mathrm{a}$ \\
\hline & 1.1 & $10.76 \mathrm{bc}$ & $10.56 \mathrm{bc}$ & $10.70 \mathrm{bc}$ & $12.42 \mathrm{a}$ & $11.11 \mathrm{a}$ & \\
\hline & 1.3 & $10.56 \mathrm{bc}$ & $9.76 \mathrm{c}-\mathrm{e}$ & $11.34 a b$ & $11.39 a b$ & $10.76 \mathrm{ab}$ & \\
\hline \multirow[t]{3}{*}{ Chahin } & 0.1 & $7.36 \mathrm{~g}$ & $7.63 \mathrm{fg}$ & $7.87 \mathrm{fg}$ & $8.61 \mathrm{e}-\mathrm{g}$ & $7.87 \mathrm{c}$ & $8.09 \mathrm{~b}$ \\
\hline & 1.1 & $7.73 \mathrm{fg}$ & $7.91 \mathrm{fg}$ & $8.90 \mathrm{~d}-\mathrm{f}$ & $8.46 \mathrm{fg}$ & $8.25 \mathrm{c}$ & \\
\hline & 1.3 & $7.87 \mathrm{fg}$ & $8.00 \mathrm{fg}$ & $8.26 \mathrm{fg}$ & $8.52 \mathrm{fg}$ & $8.16 \mathrm{c}$ & \\
\hline \multirow{2}{*}{$\begin{array}{l}\text { Hybrids } x \\
\text { Seaweed }\end{array}$} & Massouda & $10.44 \mathrm{~b}$ & $10.30 \mathrm{~b}$ & $10.70 \mathrm{~b}$ & $11.55 \mathrm{a}$ & \multirow{2}{*}{\multicolumn{2}{|c|}{ Means of compost }} \\
\hline & Chahin & $7.65 \mathrm{e}$ & $7.85 \mathrm{de}$ & $8.34 \mathrm{~cd}$ & $8.53 \mathrm{c}$ & & \\
\hline \multirow{3}{*}{$\begin{array}{l}\text { Compost } \mathbf{x} \\
\text { Seaweed }\end{array}$} & 0.1 & $8.68 \mathrm{e}$ & $9.10 \mathrm{~b}-\mathrm{e}$ & $8.97 \mathrm{c}-\mathrm{e}$ & $9.73 \mathrm{a}-\mathrm{d}$ & \multicolumn{2}{|c|}{$9.12 \mathrm{~b}$} \\
\hline & 1.1 & $9.24 b-e$ & $9.23 \mathrm{~b}-\mathrm{e}$ & 9.80 a-e & $10.44 \mathrm{a}$ & \multicolumn{2}{|c|}{$9.68 \mathrm{a}$} \\
\hline & 1.3 & $9.21 \mathrm{~b}-\mathrm{e}$ & $8.88 \mathrm{de}$ & $9.80 \mathrm{a}-\mathrm{c}$ & $9.95 \mathrm{ab}$ & \multicolumn{2}{|c|}{$9.46 \mathrm{ab}$} \\
\hline \multicolumn{2}{|c|}{ Means of seaweed } & $9.04 \mathrm{c}$ & $9.07 \mathrm{c}$ & $9.52 \mathrm{~b}$ & $10.04 \mathrm{a}$ & & \\
\hline
\end{tabular}

Means within rows or columns and their interactions followed by the same letters are not significantly differences from each other according to Duncan's multiple range test at 5\% level. 


\section{Vitamin C (\%)}

Results in the Table (3) indicated that the Massouda hybrid was showed significant increase $(3.78 \%)$ in vitamin $\mathrm{C}$ at marketable fruits than Chahin hybrid (3.28\%). Nevertheless, the level 1:1 compost observed significantly increase $(3.98 \%)$ vitamin C content in sweet pepper fruits and 0:1 compost recorded the minimum value $(3.30 \%)$. However, the influence of seaweed organic fertilizers that applied to the plants was significantly higher in two concentration $0.75-1 \mathrm{ml}^{-1}$ (Table 3) compared to untreated and $0.5 \mathrm{ml}^{-\mathrm{l}^{-1}}$ seaweed levels which measured the least results (3.21 and 3.55\%) respectively. Significant increasing in vitamin C on marketable fruits after ten days among three factors under study was found in Massouda + $1: 1$ compost and $0.75 \mathrm{ml}^{-1^{-1}}$ seaweed this interaction recorded the maximum percentage value $(4.86 \%)$ and the minimum $(2.70 \%)$ showed in Chahin, 1:3 compost and $0.0 \mathrm{ml}^{-\mathrm{l}^{-1}}$ seaweed.

\section{Fruit respiration rate $\left(\mathrm{mg} \mathrm{CO}_{2} / \mathrm{kg}-\mathrm{h}\right)$}

It noticed from the Table (4) that the respiration rates of both hybrids not recorded significant differences between them. Level of compost 1:3 revealed to significant decreases (33.74 $\mathrm{mg} \mathrm{CO} / \mathrm{kg}$-h) in fruit respiration rate than untreated plant with compost (40.46 mg $\mathrm{CO}_{2} / \mathrm{kg}-\mathrm{h}$ ). On the other hand, spraying plants by seaweed at concentration $1,0.75,0.5 \mathrm{ml}^{-1^{-1}}$ caused a significant decreasing $(33.55,35.15$ and $37.84 \mathrm{mg} \quad \mathrm{CO}_{2} / \mathrm{kg}-\mathrm{h}$ ) fruit respiration rate respectively in comparison with $0.0 \mathrm{ml}^{-\mathrm{l}^{-1}}$ seaweed treatment that had a highest value (41.25 mg CO$/ 2 / \mathrm{kg}-\mathrm{h})$. The minimum values (25.04 and $27.81 \mathrm{mg} \mathrm{CO} / \mathrm{kg}-\mathrm{h}$ ) exposed when Chahin cultivated on 1:1 compost with seaweed at 0.75 or $1 \mathrm{ml}^{-1} \mathrm{I}^{-1}$ concentration, this rates were significantly differ from most other interactions treatments, in contrast of that, the higher respiration rate $\left(49.85 \mathrm{mg} \quad \mathrm{CO}_{2} / \mathrm{kg}-\mathrm{h}\right)$ was recorded in Massouda, 1:1 compost and $0.0 \mathrm{ml}^{-1^{-}}$

seaweed.

Table (3): Effect of compost, seaweed and their interactions on vitamin C (\%) content of fruit two marketable sweet pepper hybrids after 10 days at temperature $22{ }^{\circ} \mathrm{C}$.

\begin{tabular}{|c|c|c|c|c|c|c|c|}
\hline \multirow[t]{2}{*}{ Hybrids } & \multirow{2}{*}{$\begin{array}{l}\text { Compost + } \\
\text { Soil (v/v) }\end{array}$} & \multicolumn{4}{|c|}{ Seaweed levels ml. $\mathrm{L}^{-1}$} & \multirow{2}{*}{$\begin{array}{l}\text { Hybrids } x \\
\text { Compost }\end{array}$} & \multirow{2}{*}{$\begin{array}{c}\text { Means of } \\
\text { hybrids }\end{array}$} \\
\hline & & 0 & 0.5 & 0.75 & 1 & & \\
\hline \multirow[t]{3}{*}{ Massouda } & 0.1 & $3.02 \mathrm{de}$ & $3.27 \mathrm{de}$ & $3.75 b-e$ & $3.60 \mathrm{c}-\mathrm{e}$ & $3.41 \mathrm{~b}$ & \multirow[t]{3}{*}{$3.78 \mathrm{a}$} \\
\hline & 1.1 & $4.41 \mathrm{a}-\mathrm{c}$ & $3.88 \mathrm{~b}-\mathrm{d}$ & $4.86 \mathrm{a}$ & $4.74 \mathrm{ab}$ & $4.47 \mathrm{a}$ & \\
\hline & 1.3 & $3.04 \mathrm{de}$ & $3.78 b-d$ & $3.29 \mathrm{de}$ & $3.77 b-e$ & $3.47 \mathrm{~b}$ & \\
\hline \multirow[t]{3}{*}{ Chahin } & 0.1 & $2.86 \mathrm{de}$ & $3.06 \mathrm{de}$ & $3.71 \mathrm{~b}-\mathrm{e}$ & $3.09 \mathrm{de}$ & $3.18 \mathrm{~b}$ & \multirow[t]{3}{*}{$3.28 \mathrm{~b}$} \\
\hline & 1.1 & 3.20 de & $3.84 b-d$ & 3.07 de & $3.85 b-d$ & $3.49 \mathrm{~b}$ & \\
\hline & 1.3 & $2.70 \mathrm{e}$ & $3.47 c-e$ & $3.09 \mathrm{de}$ & $3.40 c-e$ & $3.16 \mathrm{~b}$ & \\
\hline \multirow{2}{*}{$\begin{array}{l}\text { Hybrids } x \\
\text { Seaweed }\end{array}$} & Massouda & 3.49 a-d & $3.64 \mathrm{a}-\mathrm{c}$ & $3.97 \mathrm{ab}$ & $4.03 \mathrm{a}$ & \multirow{2}{*}{\multicolumn{2}{|c|}{ Means of compost }} \\
\hline & Chahin & $2.92 \mathrm{~d}$ & $3.45 b-d$ & $3.29 \mathrm{~cd}$ & $3.45 b-d$ & & \\
\hline \multirow{3}{*}{$\begin{array}{c}\text { Compost } \mathrm{x} \\
\text { Seaweed }\end{array}$} & 0.1 & $2.94 \mathrm{de}$ & $3.17 \mathrm{c}-\mathrm{e}$ & $3.73 \mathrm{a}-\mathrm{c}$ & $3.34 \mathrm{~b}-\mathrm{e}$ & \multicolumn{2}{|c|}{$3.30 \mathrm{~b}$} \\
\hline & 1.1 & $3.81 \mathrm{a}-\mathrm{c}$ & $3.86 \mathrm{a}-\mathrm{c}$ & $3.96 a b$ & $4.30 \mathrm{a}$ & \multicolumn{2}{|c|}{3.98 a } \\
\hline & 1.3 & $2.87 \mathrm{e}$ & $3.63 \mathrm{a}-\mathrm{d}$ & $3.19 \mathrm{c}-\mathrm{e}$ & 3.58 a-e & \multicolumn{2}{|c|}{$3.32 b$} \\
\hline \multicolumn{2}{|c|}{ Means of seaweed } & $3.21 \mathrm{~b}$ & $3.55 \mathrm{ab}$ & $3.63 \mathrm{a}$ & $3.74 \mathrm{a}$ & & \\
\hline
\end{tabular}

Means within rows or columns and their interactions followed by the same letters are not significantly differences from each other according to Duncan's multiple range test at $5 \%$ level.

Table (4): Effect of compost, seaweed and their interactions on fruit respiration rate ( $\left.\mathrm{mg} \mathrm{CO}_{2} / \mathrm{kg}-\mathrm{h}\right)$ of two marketable sweet pepper hybrids after 10 days at temperature $22{ }^{\circ} \mathrm{C}$.

\begin{tabular}{|c|c|c|c|c|c|c|c|}
\hline \multirow[t]{2}{*}{ Hybrids } & \multirow{2}{*}{$\begin{array}{l}\text { Compost + } \\
\text { Soil }(v / v)\end{array}$} & \multicolumn{4}{|c|}{ Seaweed levels ml. $\mathrm{L}^{-1}$} & \multirow{2}{*}{$\begin{array}{l}\text { Hybrids } x \\
\text { Compost }\end{array}$} & \multirow{2}{*}{$\begin{array}{l}\text { Means of } \\
\text { hybrids }\end{array}$} \\
\hline & & 0 & 0.5 & 0.75 & 1 & & \\
\hline Massouda & 0.1 & $47.31 \mathrm{fg}$ & $44.37 \mathrm{e}-\mathrm{g}$ & $41.85 \mathrm{c}-\mathrm{g}$ & $33.40 \mathrm{a}-\mathrm{d}$ & $41.73 \mathrm{c}$ & $40.80 \mathrm{a}$ \\
\hline
\end{tabular}




\begin{tabular}{|c|c|c|c|c|c|c|}
\hline & 1.1 & $49.85 \mathrm{~g}$ & $37.53 \mathrm{~b}-\mathrm{e}$ & $47.02 \mathrm{fg}$ & $41.46 \mathrm{c}-\mathrm{g}$ & $43.96 \mathrm{c}$ \\
\hline & 1.3 & $38.42 b-f$ & $39.56 \mathrm{~b}-\mathrm{g}$ & $31.47 \mathrm{ab}$ & $37.33 \mathrm{~b}-\mathrm{e}$ & $36.69 \mathrm{a}-\mathrm{c}$ \\
\hline \multirow[t]{3}{*}{ Chahin } & 0.1 & $46.11 \mathrm{e}-\mathrm{g}$ & $42.28 \mathrm{~d}-\mathrm{g}$ & 37.66 b-e & $30.68 \mathrm{ab}$ & $39.18 \mathrm{bc}$ \\
\hline & 1.1 & $32.79 \mathrm{a}-\mathrm{c}$ & $31.62 \mathrm{ab}$ & $25.04 \mathrm{a}$ & $27.81 \mathrm{a}$ & $29.31 \mathrm{a}$ \\
\hline & 1.3 & $33.02 \mathrm{a}-\mathrm{d}$ & $31.66 \mathrm{ab}$ & $27.87 \mathrm{a}$ & $30.60 \mathrm{ab}$ & $30.79 \mathrm{ab}$ \\
\hline \multirow{2}{*}{$\begin{array}{l}\text { Hybrids } x \\
\text { Seaweed }\end{array}$} & Massouda & $45.20 \mathrm{~d}$ & $40.49 \mathrm{c}$ & $40.11 \mathrm{bc}$ & $37.39 \mathrm{bc}$ & \multirow[t]{2}{*}{ Means of compost } \\
\hline & Chahin & $37.31 \mathrm{bc}$ & $35.18 \mathrm{~b}$ & $30.19 \mathrm{a}$ & $29.70 \mathrm{a}$ & \\
\hline \multirow{3}{*}{$\begin{array}{l}\text { Compost } \mathrm{x} \\
\text { Seaweed }\end{array}$} & 0.1 & $46.71 \mathrm{e}$ & $43.33 \mathrm{de}$ & $39.75 \mathrm{~b}-\mathrm{d}$ & $32.04 \mathrm{a}$ & $40.46 \mathrm{~b}$ \\
\hline & 1.1 & 41.32 c-e & $34.57 \mathrm{ab}$ & $36.03 \mathrm{a}-\mathrm{c}$ & $34.64 \mathrm{ab}$ & $36.64 \mathrm{ab}$ \\
\hline & 1.3 & $35.72 \mathrm{a}-\mathrm{c}$ & $35.61 \mathrm{a}-\mathrm{c}$ & $29.67 \mathrm{a}$ & $33.97 \mathrm{ab}$ & $33.74 \mathrm{a}$ \\
\hline \multicolumn{2}{|c|}{ Means of seaweed } & $41.25 \mathrm{c}$ & $37.84 \mathrm{~b}$ & $35.15 a b$ & $33.55 \mathrm{a}$ & \\
\hline
\end{tabular}

Means within rows or columns and their interactions followed by the same letters are not significantly differences from each other according to Duncan's multiple range test at 5\% level.

\section{Carbohydrate contents (\%)}

It is obvious from the Table (5) that the both hybrids did not show any significant difference in total carbohydrate content of marketable fruits. The fruit of 1:1 compost increased total carbohydrate content (14.88\%) in fruits, followed 1:3 compost that has (13.54\%) compared to untreated plant with compost. On the other hand, the application of seaweed was valuable significant resulted on the fruit carbohydrate, hence $1 \mathrm{ml}^{-1^{-1}}$ seaweed recorded the higher value $(15.32 \%)$ and $0.0 \mathrm{ml} . \mathrm{l}^{-1}$ seaweed recorded the lowest $(11.88 \%)$ value. Results in table (5) indicated that the effects of the interaction among three factors were significantly recorded on the percentage of carbohydrate in fruits, when the Massouda cultivated with 0:1 compost and sprayed with 1 ml. $\mathrm{l}^{-1}$ seaweed extract gave the best percentage value (22.07) and the lowest rate (6.54\%) noticed in Massouda, 0:1 compost and $0.0 \mathrm{ml}^{-\mathrm{l}^{-1}}$ seaweed.

Table (5): Effect of compost, seaweed and their interactions on total carbohydrate (\%) content of two marketable sweet pepper hybrids after 10 days at temperature $22{ }^{\circ} \mathrm{C}$.

\begin{tabular}{|c|c|c|c|c|c|c|c|}
\hline \multirow[t]{2}{*}{ Hybrids } & \multirow{2}{*}{$\begin{array}{c}\text { Compost + } \\
\text { Soil (v/v) }\end{array}$} & \multicolumn{4}{|c|}{ Seaweed levels ml.L-1 } & \multirow{2}{*}{$\begin{array}{l}\text { Hybrids } \mathrm{X} \\
\text { Compost }\end{array}$} & \multirow{2}{*}{$\begin{array}{l}\text { Means of } \\
\text { hybrids }\end{array}$} \\
\hline & & $\mathbf{0}$ & 0.5 & 0.75 & 1 & & \\
\hline \multirow[t]{3}{*}{ Massouda } & 0.1 & $6.54 \mathrm{~g}$ & $9.04 \mathrm{fg}$ & $17.11 \mathrm{a}-\mathrm{e}$ & $22.07 \mathrm{a}$ & $13.69 \mathrm{~b}$ & \multirow[t]{3}{*}{$16.36 \mathrm{a}$} \\
\hline & 1.1 & $16.63 \mathrm{a}-\mathrm{e}$ & $20.60 a b$ & $18.44 \mathrm{a}-\mathrm{d}$ & $18.29 \mathrm{a}-\mathrm{d}$ & $18.49 \mathrm{a}$ & \\
\hline & 1.3 & $19.30 \mathrm{a}-\mathrm{c}$ & $21.37 \mathrm{a}$ & $13.17 \mathrm{c}-\mathrm{g}$ & $13.75 b-f$ & $16.90 \mathrm{a}$ & \\
\hline \multirow[t]{3}{*}{ Chahin } & 0.1 & $7.11 \mathrm{fg}$ & $8.19 \mathrm{fg}$ & $18.09 \mathrm{a}-\mathrm{d}$ & $11.67 \mathrm{~d}-\mathrm{g}$ & $11.26 \mathrm{bc}$ & \multirow[t]{3}{*}{$10.90 \mathrm{a}$} \\
\hline & 1.1 & $11.06 \mathrm{e}-\mathrm{g}$ & $6.88 \mathrm{fg}$ & $13.71 b-f$ & $13.42 \mathrm{c}-\mathrm{g}$ & $11.27 \mathrm{bc}$ & \\
\hline & 1.3 & $10.61 \mathrm{e}-\mathrm{g}$ & $8.65 \mathrm{fg}$ & $8.73 \mathrm{fg}$ & $12.71 \mathrm{c}-\mathrm{g}$ & $10.18 \mathrm{c}$ & \\
\hline \multirow{2}{*}{$\begin{array}{l}\text { Hybrids } \mathrm{X} \\
\text { Seaweed }\end{array}$} & Massouda & $14.16 \mathrm{bc}$ & $17.00 \mathrm{ab}$ & $16.24 \mathrm{a}-\mathrm{c}$ & $18.04 \mathrm{a}$ & \multirow{2}{*}{\multicolumn{2}{|c|}{ Means of compost }} \\
\hline & Chahin & $9.59 \mathrm{de}$ & $7.90 \mathrm{e}$ & $13.51 \mathrm{bc}$ & $12.60 \mathrm{~cd}$ & & \\
\hline \multirow{3}{*}{$\begin{array}{c}\text { Compost } x \\
\text { Seaweed }\end{array}$} & 0.1 & $6.82 \mathrm{c}$ & $8.61 \mathrm{c}$ & $17.60 \mathrm{a}$ & $16.87 \mathrm{a}$ & \multicolumn{2}{|c|}{$12.48 \mathrm{~b}$} \\
\hline & 1.1 & $13.85 \mathrm{ab}$ & $13.74 \mathrm{ab}$ & $16.08 \mathrm{a}$ & $15.85 \mathrm{a}$ & \multicolumn{2}{|c|}{$14.88 \mathrm{a}$} \\
\hline & 1.3 & $14.96 \mathrm{ab}$ & $15.01 \mathrm{ab}$ & $10.95 \mathrm{bc}$ & $13.23 \mathrm{ab}$ & \multicolumn{2}{|c|}{$13.54 \mathrm{ab}$} \\
\hline \multicolumn{2}{|c|}{ Means of seaweed } & $11.88 \mathrm{c}$ & $12.45 b c$ & $14.87 a b$ & $15.32 \mathrm{a}$ & & \\
\hline
\end{tabular}

Means within rows or columns and their interactions followed by the same letters are not significantly differences from each other according to Duncan's multiple range test at $5 \%$ level. 
Chlorophyll a content in fruits (mg.g $\left.{ }^{-1}\right)$

The results obtained from Table (6) revealed that the Chahin hybrid was significantly showed higher chlorophyll a (1.31 $\left.\mathrm{mg}^{\mathrm{g}} \mathrm{g}^{-1}\right)$ after marketable condition in compared to Massouda $\left(1.05 \mathrm{mg} \cdot \mathrm{g}^{-1}\right)$. On the other hand, the maximum $\left(1.34 \mathrm{mg} . \mathrm{g}^{-1}\right)$ significant chlorophyll a resulted from the effect of compost in 1:3 level and the least $\left(1.08 \mathrm{mg} . \mathrm{g}^{-1}\right)$ was obtained in control. Seaweed fertilizers that applied to the plants recorded the best chlorophyll a $\left(1.24 \mathrm{mg} \cdot \mathrm{g}^{-1}\right)$ at concentration $0.5 \mathrm{ml}^{-\mathrm{l}^{-1}}$ and the minimum (1.13 mg. $\mathrm{g}^{-1}$ ) was found in untreated plants. After 10 days of marketable, pepper fruits had significant increases chlorophyll a $\left(1.66 \mathrm{mg} \cdot \mathrm{g}^{-1}\right)$ return to the interaction among Chahin hybrid when planted without compost and sprayed with $0.5 \mathrm{ml}^{-1^{-1}}$ seaweed, whereas the lowest value $\left(0.73 \mathrm{mg} \cdot \mathrm{g}^{-1}\right)$ recorded in Massouda, compost 1:1 and $0.5 \mathrm{ml}^{-1^{-}}$ ${ }^{1}$ seaweed.

\section{Chlorophyll b content in fruits ( $\mathrm{mg.g}^{-1}$ )}

Data in the Table (7) indicated that Chahin hybrid was significantly improved fruit chlorophyll b (0.42 mg.g $\left.\mathrm{g}^{-1}\right)$ than Massouda hybrid $\left(0.22 \mathrm{mg} \cdot \mathrm{g}^{-1}\right)$. However, the influence of compost was not observed significant increase in fruit chlorophyll b. whereas seaweed at concentration $1 \mathrm{ml}^{-\mathrm{l}^{-1}}$ recorded significantly the maximum rate $\left(0.42 \mathrm{mg} \cdot \mathrm{g}^{-1}\right)$ but there were no significant differences between the other levels and control, hence the lowest value was recorded in the rest of levels. The combination of the three factors (hybrids, compost and seaweed) has a profound significant effect on chlorophyll $b$ content in marketable sweet pepper fruits, the best value $\left(0.63 \mathrm{mg} \cdot \mathrm{g}^{-1}\right)$ showed from Chahin, 0:1 compost and treated with $1 \mathrm{ml}^{-1} \mathrm{l}^{-1}$ seaweed, whereas a minimum value $\left(0.04 \mathrm{mg} \cdot \mathrm{g}^{-1}\right)$ was showed in Massouda hybrid which planted without compost and treated with seaweed at concentration

Table (6): Effect of compost, seaweed and their interactions on chlorophyll a $\left(\mathrm{mg} \cdot \mathrm{g}^{-1}\right)$ content in two marketable sweet pepper hybrids after 10 days at temperature $22^{\circ} \mathrm{C}$.

\begin{tabular}{|c|c|c|c|c|c|c|c|}
\hline \multirow[t]{2}{*}{ Hybrids } & \multirow{2}{*}{$\begin{array}{c}\text { Compost + } \\
\text { Soil (v/v) }\end{array}$} & \multicolumn{4}{|c|}{ Seaweed levels ml. $\mathrm{L}^{-1}$} & \multirow{2}{*}{$\begin{array}{l}\text { Hybrids } x \\
\text { Compost }\end{array}$} & \multirow{2}{*}{$\begin{array}{c}\text { Means } \\
\text { of } \\
\text { hybrids }\end{array}$} \\
\hline & & 0 & 0.5 & 0.75 & 1 & & \\
\hline \multirow[t]{3}{*}{ Massouda } & 0.1 & $0.94 \mathrm{~g}-\mathrm{i}$ & $0.83 \mathrm{hi}$ & $1.05 \mathrm{f}-\mathrm{h}$ & $0.96 \mathrm{~g}-\mathrm{i}$ & $0.94 \mathrm{c}$ & $1.05 \mathrm{~b}$ \\
\hline & 1.1 & $1.42 \mathrm{a}-\mathrm{c}$ & $0.73 \mathrm{i}$ & $0.90 \mathrm{~g}-\mathrm{i}$ & $0.84 \mathrm{hi}$ & $0.97 \mathrm{bc}$ & \\
\hline & 1.3 & $0.98 \mathrm{~g}-\mathrm{i}$ & $1.32 b-e$ & $1.16 \mathrm{e}-\mathrm{g}$ & $1.43 \mathrm{a}-\mathrm{c}$ & $1.22 \mathrm{ab}$ & \\
\hline \multirow[t]{3}{*}{ Chahin } & 0.1 & $0.81 \mathrm{hi}$ & $1.66 \mathrm{a}$ & $1.41 \mathrm{a}-\mathrm{d}$ & $0.98 \mathrm{~g}-\mathrm{i}$ & $1.21 \mathrm{a}-\mathrm{c}$ & $1.31 \mathrm{a}$ \\
\hline & 1.1 & $1.13 \mathrm{e}-\mathrm{g}$ & $1.44 \mathrm{a}-\mathrm{c}$ & $0.94 \mathrm{~g}-\mathrm{i}$ & $1.50 \mathrm{a}-\mathrm{c}$ & $1.25 \mathrm{a}$ & \\
\hline & 1.3 & $1.51 \mathrm{a}-\mathrm{c}$ & $1.49 \mathrm{a}-\mathrm{c}$ & $1.58 \mathrm{ab}$ & $1.25 c-f$ & $1.46 \mathrm{a}$ & \\
\hline \multirow{2}{*}{$\begin{array}{l}\text { Hybrids } x \\
\text { Seaweed }\end{array}$} & Massouda & $1.11 \mathrm{~cd}$ & $0.96 \mathrm{e}$ & $1.03 \mathrm{de}$ & $1.08 \mathrm{de}$ & \multirow{2}{*}{\multicolumn{2}{|c|}{ Means of compost }} \\
\hline & Chahin & $1.15 \mathrm{~cd}$ & $1.53 \mathrm{a}$ & $1.31 \mathrm{~b}$ & $1.24 \mathrm{bc}$ & & \\
\hline \multirow{3}{*}{$\begin{array}{c}\text { Compost } \mathrm{x} \\
\text { Seaweed }\end{array}$} & 0.1 & $0.87 \mathrm{e}$ & $1.24 \mathrm{a}-\mathrm{c}$ & $1.23 \mathrm{a}-\mathrm{c}$ & $0.97 \mathrm{de}$ & \multicolumn{2}{|c|}{$1.08 \mathrm{~b}$} \\
\hline & 1.1 & $1.28 \mathrm{ab}$ & $1.08 \mathrm{~cd}$ & $0.92 \mathrm{de}$ & $1.17 \mathrm{bc}$ & \multicolumn{2}{|c|}{$1.11 \mathrm{~b}$} \\
\hline & 1.3 & $1.25 \mathrm{a}-\mathrm{c}$ & $1.40 \mathrm{a}$ & $1.37 \mathrm{a}$ & $1.34 \mathrm{ab}$ & \multicolumn{2}{|c|}{$1.34 \mathrm{a}$} \\
\hline \multicolumn{2}{|c|}{ Means of seaweed } & $1.13 \mathrm{~b}$ & $1.24 \mathrm{a}$ & $1.17 \mathrm{ab}$ & $1.16 \mathrm{ab}$ & & \\
\hline
\end{tabular}

Means within rows or columns and their interactions followed by the same letters are not significantly differences from each other according to Duncan's multiple range test at 5\% level. 
Table (7): Effect of compost, seaweed and their interactions on chlorophyll b (mg.g $\mathrm{g}^{-1}$ ) content in two marketable sweet pepper hybrids after 10 days at temperature $22^{\circ} \mathrm{C}$.

\begin{tabular}{|c|c|c|c|c|c|c|c|}
\hline \multirow[t]{2}{*}{ Hybrids } & \multirow{2}{*}{$\begin{array}{c}\text { Compost + } \\
\text { Soil (v/v) }\end{array}$} & \multicolumn{4}{|c|}{ Seaweed levels ml. $\mathrm{L}^{-1}$} & \multirow{2}{*}{$\begin{array}{l}\text { Hybrids } \mathrm{x} \\
\text { Compost }\end{array}$} & \multirow{2}{*}{$\begin{array}{c}\text { Means } \\
\text { of } \\
\text { hybrids }\end{array}$} \\
\hline & & 0 & 0.5 & 0.75 & 1 & & \\
\hline \multirow[t]{3}{*}{ Massouda } & 0.1 & 0.16 ef & $0.04 \mathrm{f}$ & $0.31 b-f$ & $0.25 c-f$ & $0.19 \mathrm{~b}$ & $0.22 \mathrm{~b}$ \\
\hline & 1.1 & 0.15 ef & $0.31 \mathrm{~b}-\mathrm{f}$ & 0.17 ef & $0.26 \mathrm{c}-\mathrm{f}$ & $0.22 \mathrm{~b}$ & \\
\hline & 1.3 & $0.37 \mathrm{a}-\mathrm{e}$ & $0.23 \mathrm{c}-\mathrm{f}$ & $0.21 \mathrm{~d}-\mathrm{f}$ & 0.17 ef & $0.25 \mathrm{~b}$ & \\
\hline \multirow[t]{3}{*}{ Chahin } & 0.1 & $0.22 c-f$ & $0.51 \mathrm{a}-\mathrm{c}$ & $0.37 \mathrm{a}-\mathrm{e}$ & $0.63 \mathrm{a}$ & $0.43 \mathrm{a}$ & $0.42 \mathrm{a}$ \\
\hline & 1.1 & $0.47 \mathrm{a}-\mathrm{d}$ & $0.30 \mathrm{c}-\mathrm{f}$ & $0.26 c-f$ & $0.60 \mathrm{a}$ & $0.41 \mathrm{a}$ & \\
\hline & 1.3 & $0.36 \mathrm{a}-\mathrm{e}$ & $0.25 c-f$ & $0.42 \mathrm{a}-\mathrm{e}$ & $0.59 a b$ & $0.41 \mathrm{a}$ & \\
\hline \multirow{2}{*}{$\begin{array}{l}\text { Hybrids } x \\
\text { Seaweed }\end{array}$} & Massouda & $0.23 \mathrm{~b}$ & $0.19 \mathrm{~b}$ & $0.23 \mathrm{~b}$ & $0.23 \mathrm{~b}$ & \multirow{2}{*}{\multicolumn{2}{|c|}{ Means of compost }} \\
\hline & Chahin & $0.35 \mathrm{~b}$ & $0.35 \mathrm{~b}$ & $0.35 \mathrm{~b}$ & $0.61 \mathrm{a}$ & & \\
\hline \multirow{3}{*}{$\begin{array}{c}\text { Compost x } \\
\text { Seaweed }\end{array}$} & 0.1 & $0.19 \mathrm{~b}$ & $0.28 a b$ & $0.34 a b$ & $0.44 \mathrm{a}$ & \multicolumn{2}{|c|}{$0.31 \mathrm{a}$} \\
\hline & 1.1 & $0.31 a b$ & $0.31 a b$ & $0.21 \mathrm{~b}$ & $0.43 \mathrm{a}$ & \multicolumn{2}{|c|}{$0.32 \mathrm{a}$} \\
\hline & 1.3 & $0.37 \mathrm{ab}$ & $0.24 \mathrm{ab}$ & $0.32 a b$ & $0.38 \mathrm{ab}$ & \multicolumn{2}{|c|}{$0.33 \mathrm{a}$} \\
\hline \multicolumn{2}{|c|}{ Means of seaweed } & $0.29 \mathrm{~b}$ & $0.27 \mathrm{~b}$ & $0.29 \mathrm{~b}$ & $0.42 \mathrm{a}$ & & \\
\hline
\end{tabular}

Means within rows or columns and their interactions followed by the same letters are not significantly differences from each other according to Duncan's multiple range test at 5\% level.

\section{DISCUSSION}

It is observed from the above mentioned results in Tables $(2,3,6$ and 7$)$ that a significant increase occurred in fruit dry weight, vitamin $\mathrm{C}$, chlorophyll a and b. Increasing fruit dry weight and vitamin $\mathrm{C}$ showed in Massouda hybrid while increasing chlorophyll $\mathrm{a}$ and $\mathrm{b}$ in fruits showed in Chahin hybrid, this could be attributed to genetic capacity of both hybrids and causes highly differences in terms of fruits quality, growth and development parameters of pepper plants which largely depend on the genetic constitutions of the plants (Ganiger, 2010), results of the present study are in agreement with (Karanatsidis and Berova 2009). However, fruit parameters were significantly influenced by hybrids for example; dry matter content of sweet pepper fruits differed significantly between the cultivars (Jadczak et al., 2010).

The change in fruit dry weight, vitamin $\mathrm{C}$, fruit respiration, carbohydrate and chlorophyll a in sweet pepper due to the compost is presented in the Tables $(2,3,4,5$, and 6) respectively. The effects of compost alone on the fruit quality were showed significantly better than untreated treatments in the most above mentioned characters. Our results shows at high level of compost significantly increased fruit dry weight, vitamin $\mathrm{C}$ and carbohydrate content. Similarly, results of (Peyvast $\boldsymbol{e t}$ al., 2007) they proved that compost treatments at high amount, significantly influenced dry weight of green pepper, vitamin $\mathrm{C}$ content, chlorophyll $\mathrm{a}$ and $\mathrm{b}$ in fruits. Also agree with those observed by (Copetta et al., 2011 and Aminifard et al., 2013) who determined that compost application at different levels improved carbohydrate content, and with those (Piazzolla et al., 2012) in fruit respiration at compost levels compared to other treatments, this might be due to increasing compost in soil, the level of macronutrients were increased significantly therefore chlorophyll pigments and vitamin $\mathrm{C}$ synthesis increased with macronutrients increased in the soil (Zhao et al., 2005). On the other hand, vitamin $C$ levels in vegetables depend on several factors, including cultivars, plant nutrition, production practice and maturity (Antonio et al., 2007).

The results revealed that foliar spray of seaweed significantly influenced fruit parameters. Foliar spray of seaweed along enhanced all fruits parameters including fruit dry weight, vitamin $\mathrm{C}$, carbohydrate, chlorophyll a, $\mathrm{b}$ and decreasing fruit respiration rate. Seaweed affected the structure of plant roots and facilitating efficient uptake of nutrients either through roots or from the leaf surface 
(Battacharyya et al., 2015). Vijayakumar et al., (2018) reported that lower concentration of seaweed liquid fertilizer has stimulated chlorophyll and carbohydrate content in sweet pepper as compared to control, this influence might be due to more availability of plant nutrients and growth promoting hormones which ultimately resulted in the higher values of the growth and fruits parameters, because the seaweed extracts have growth stimulating activities and are used as bio-stimulants in natural crop protection (Vinoth et al., 2012). Also it was reported that the seaweed extracts have promising plant growth regulators such as Auxins, cytokinins, gibberellins, betains and major macronutrients and micronutrients which help in promoting the growth of various vegetables, fruits and other crops (Blunden $\boldsymbol{e t}$ al., 1991). Our results are in harmony with those observed by (Vijayakumar et al., 2018).

\section{Conclusions}

The obtained results cleared that the fruit marketable and quality of sweet pepper tested in the experiment significantly showed different between hybrids. Although there were significant increases between compost levels alone on fruit dry weight, vitamin C, carbohydrate, chlorophyll a and lower fruit respiration rate with comparison to control except chlorophyll b. However, the highest rates of all parameters in fruit marketability were found in hybrids when treated with high concentration of seaweed extract, also the positive effect of the compost and seaweed extract together on the pepper was expressed in both hybrids by improving all characters compared to control.

\section{REFERENCE}

A.O.A.C. International. (2000). Official Methods of Analysis. 11th Edition Washington D.C. Association of Official Analysis Chemist. P. 1015.

Adediran, J.A.; L.B. Taiwo and R.A. Sobulo. (2003). Effect of Organic Wastes and Method of Composting on Compost Maturity, Nutrient Composition of Compost and Yields of Two Vegetable Crops, Journal of Sustainable Agriculture, 22:4, 95-109.
Adhikari, P.; A. Khanal and R. Subedi. (2016). Effect of Different Sources of Organic Manure on Growth and Yield of Sweet Pepper. Adv Plants Agric Res 3(5).

Al-any, A.M. (1985). Post-Harvest physiology of horticultural crops. University of Baghdad, Mosel press. The Republic of Iraq. (In Arabic).

Aminifard, M.; H. Aroiee, M. Azizi, H. Nemati, and H. Jaafar. (2013). Effect of compost on antioxidant components and fruit quality of sweet pepper (Capsicum annuиm L.). Journal of Central European Agriculture, 14(2), 0-0.

Anonymous. (1989). Tomato and Pepper Production in the Tropics. Asian. Vegetable Research and Development Center, Taiwan. p585.

Antonio, J.P.; M.A Francisco, S.M. Ana, I.F. Maria, and D. Estrella. (2007). Influence of agricultural practices on the quality of sweet pepper fruits as affected by the maturity stage. Journal of the Science of Food and Agriculture, 87, 2075 - 2080.

Arancon, N.Q.; C.A. Edward, R.M. Atiyeh, and J.D. Metzger. (2004) Effect of vermicomposts produced from cattle manure, food waste and paper waste on the growth and yield of peppers in the field. Pedobiologia, 49, 297306.

Arumugam, R. and P. Anantharaman. (2009). Effect of Seaweed Liquid Fertilizer on Growth and Pigment Concentration of Abelmoschus esculentus (1) medikus. Journal of Agronomy 2 (2): 57-66.

Battacharyya, D.; M.Z. Babgohari, P. Rathor, and B. Prithiviraj. (2015). Seaweed extracts as biostimulants in horticulture. Scientia Horticulturae, 196, 39-48.

Baysal, E.; O.N. Yigitbasi, M. Colak, H. Toker, H. Simsek, and F. Yilmaz. (2007). Cultivation of Agaricus bisporus on some compost formulas and locally available casing materials. Part I: Wheat straw based compost formulas and locally available casing materials. African 
Journal of Biotechnology Vol. 6 (19), pp. 2225-2230.

Blunden, G.; B.E. Smith, T.G. Mason, X.U. Ying, J.C. Yvin, and R. Chabot. (1991). Betaines in seaweed extracts. J Eur Cosmet 13:113-118.

CASFS. (2017). A Project for the Center of Agroecology and Sustainable Food Systems. University of California, Santa Cruz. Unit 1.7. Making and Using. Compost, (casfs.ucsc.edu).

Cheng, Y.; Y. Zheng, C. Tai, J. Yen, Y. Chen, and F. Jan. (2014). Identification, characterisation and detection of a new tospovirus on sweet pepper. Annals of Applied Biology, 164(1), 107-115.

Copetta, A.; L. Bardi, E. Bertolone, and G. Berta. (2011). Fruit production and quality of tomato plants (Solanum lycopersicum L.) are affected by green compost and arbuscular mycorrhizal fungi. Plant biosystems, 145(1), 106-115.

Compost Management Programs. A. and L. Canada Laboratories Inc. (2004). Compost Analysis for Available Nutrients and Soil Suitability Criteria and Evaluation. 2136 Jetstream Road. London, Ontario, N5V 3P5. P, 6.

Duffy, B.; S. Ravva, and C. Sarreal. (2013). Effect of Molasses on Regrowth of E. coli O157:H7 and Salmonella in Compost Teas. Compost Science and Utilization, Vol. 12, No. 1, 93-96.

Epstein, E. (2011). Industrial Composting, Environmental Engineering and Facilities Management. CRC Press is an imprint of the Taylor and Francis Group. Boca Raton London New York. Pp, 26_27.

Ganiger, V.M. (2010). Response of bell pepper to organic nutrition under different environments. Thesis submitted to the University of Agricultural Sciences, Dharwad.

Gobara, A.A. (1998). Response of 'Le-Conte' pear trees to foliar applications of some nutrients. Egypt. J. Hort. 25(1): 55-70.
Hallmann, E.R. and E. Rembialkowska. (2012). Characterisation of antioxidant compounds in sweet bell pepper (Capsicum annuum L.) under organic and conventional growing systems. Journal of the Science of Food and Agriculture, Vol. 92(12), pp. 2409-2415.

Jadczak, D.; M. Grzeszezuk, and D. Kosecka. (2010). Quality characteristics and content of mineral compounds in fruit of some cultivars of sweet pepper (Capsicum annuиm L.). J. Elementol. 15(3): 509-515.

Joslyn, M.A. (1970). Methods in Food Analysis (2). Acad. Press, N. Y. London.

Karanatsidis, G.; and M. Berova. (2009). Effect of Organic-N Fertilizer on Growth and Some Physiological Parameters in Pepper Plants (Capsicum annuum L.). Biotechnology and Biotechnological Equipment, 23, 254-257.

Khan, N.; I. Clark, M.A. Sanchez-Monedero, S. Shea, S. Meier, and N. Bolan. (2014). Maturity indices in co-composting of chicken manure and sawdust with biochar. Bioresource Technology 168, 245-251.

Llaven, M.A.O.; J.L.G. Jimenez, B.I.C. Coro, R. Rincon-Rosales, J.M. Molina, L. Dendooven, and A.G.M. Federico. (2008). Fruit Characteristics of Bell Pepper Cultivated in Sheep Manure Vermicompost Substituted Soil, Journal of Plant Nutrition, 31(9), 15851598.

Manna, D.; A. Sarkar, and T.K. Maity. (2012). Impact of biozyme on growth, yield andquality of chilli (Capsicum annuum L.). J. Crop Weed 8, 40-43.

Peyvast, G.H.; M.S. Moghaddam, and J.A. Olfati. (2007). Effect of municipal solid waste compost on weed control, yield and some quality indices of green pepper (Capsicum annuum L.). Biosci. Biotechnol. Res. Asia, 4(2), 449-456.

Piazzolla, F.; M.L. Amodio, R. Rinaldi, F. Raimo, and G. Colelli. (2012). Effect of type of fertilization and maturity on quality of fresh- 
cut red and yellow peppers (Capsicum annuum L.). In II International Conference on Quality Management of Fresh Cut Produce: Convenience Food for a Tasteful Life 1209. pp. 391-398.

Raffo, A.; I. Baiamonte, N. Nardo, and F. Paoletti. (2007). Internal quality and antioxidants content of cold-stored red sweet peppers as affected by polyethylene bag packaging and host water treatment. Eur Food Res Technol 225:395-405.

Rittenhouse, T. (2015). Tipsheet: Compost. Produced by the National Center for Appropriate Technology. www.attra.ncat.org. U.S. Department of Agriculture's Agricultural Marketing Service, National Organic Program. 1-800-275-6228. Pp. 2-4.

Rubatzky, V. and M. Yamaguchi. (1997). World Vegetables: Principles, Production, and Nutritive Values (2nd ed.). New York: Chapman and Hall.

Safford, W.E. (1926). Our heritage from the American Indians. Journal of the Illinois State Archaeological Society 4(1): 405-410
Production in Vegetable Crops 607.pp. 125128.

United States Agency for International DevelopmentInma. (USAID). (2011-2012). Pepper Production in Iraq. Agribusiness team for a consortium led by The Louis Berger Group, Inc. p31. Website: www.inma-iraq.com.

Vijayakumar, S.; S. Durgadevi, P. Arulmozhi, S. Rajalakshmi, T. Gopalakrishnan, and N. Parameswari. (2018). Effect of seaweed liquid fertilizer on yield and quality of Capsicum annuиm L. Acta Ecologica Sinica.

Vinoth, S.; P. Gurusaravanan, and N. Jayabalan. (2012). Effect of seaweed extracts and plant growth regulators on high-frequency in vitro mass propagation of Lycopersicon esculentum L (tomato) through double cotyledonary nodal explant. Journal of applied phycology, 24(5), $1329-1337$

Wintermans, J.F.G.M. and A. DeMots. (1965). Spectrophotometric characteristics of chlorophyll $\mathrm{a}$ and $\mathrm{b}$ and their phynophytins in ethanol. Biochem. Biophys. Acta, 109: 448453.

Sasikala, M.; E. Indumathi, S. Radhika, anWoRmann, C.S. and C.A. Shapiro. (2012). Composting Manure Sasireka. (2016). Effect of Seaweed Extract (Sargassum tenerrimum) on Seed Germination and growth of Tomato Plant. International Journal of ChemTech Research. Vol.9, No.09 pp 285-293.

Zaki, N.; H. Aziz, C.E. Kaoutar, and D. Fatima. (2017).

Stirk, W.A.; G.D. Arthur, A.F. Lourens, O. Novak, M. Strnad, and J. Van-Staden. (2004). Changes in cytokinin and auxin concentrations in seaweed concentrates when stored at an elevated temperature. J. Appl Phycol 16:3139.

Stoffella, P.J.; M. Ozores-Hampton, N.E. Roe, Zhњi், D.; K.R. Reddy, and V.R. Reddy. (2005). Nitrogen and T.A. Obreza. (2003). Compost utilization in vegetable crop production systems. In IX International Symposium on Timing of Field and Other Organic Materials. University of NebraskaLincoln Extension, Institute of agriculture and natural resources, United State, Department of Agriculture. G1315. Pp.4.

Comparative Evaluation of the Phytochemical Constituents and the Antioxidant Activities of Five Moroccan Pepper Varieties (Capsicum annuum L.). Ouatmane J. Chem. Bio. Phy. Sci. Sec. B, Vol. 7, No. 4, 1294-1306. deficiency effects on plant growth, leaf photosynthesis, and hyper spectral reflectance properties of sorghum. European Journal of Agronomy.

22:

391-403. 
كارتيّكرنا تيّكه لهى وگياينّ دهريايى لسهر ساخلهتيّن فيزيولوجى يِين دوو جوريّن ههجين ييّن فلفلا

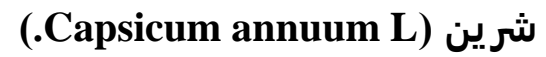

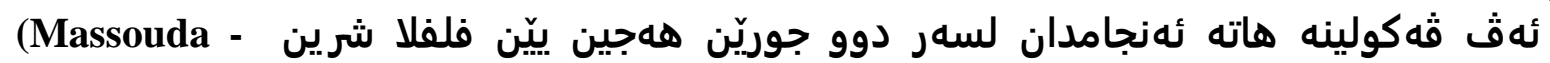
بـو Chahin) (Capsicum annuum L.)

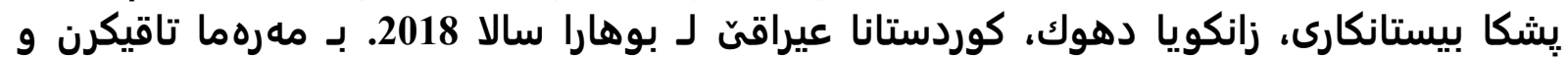

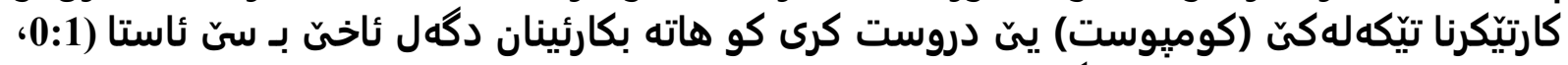

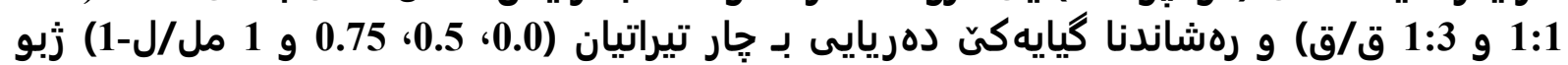

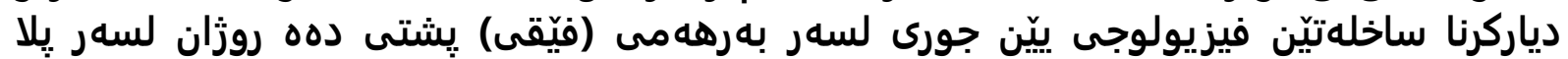

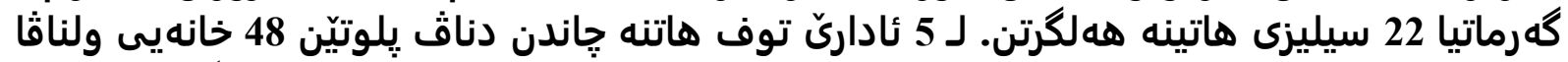

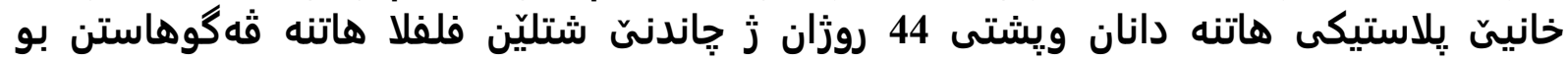

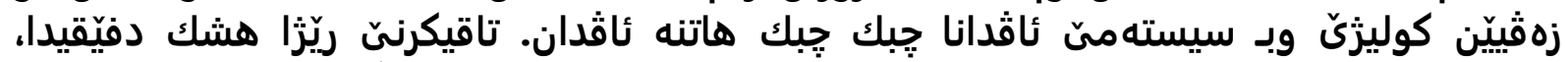

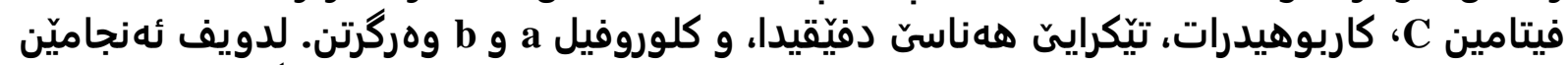

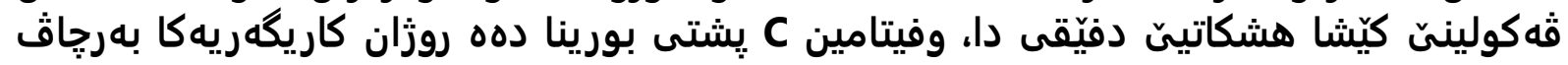

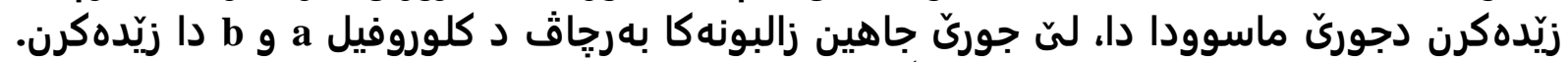

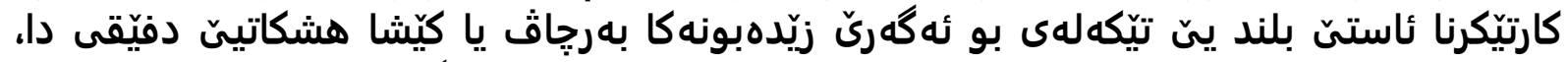

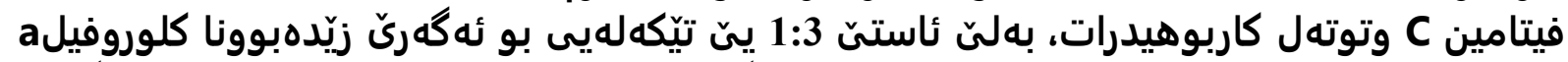

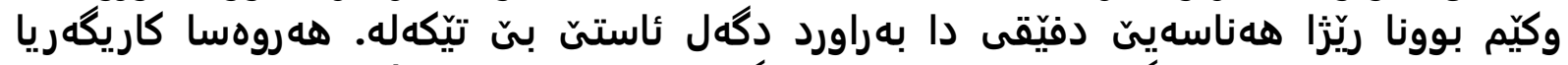

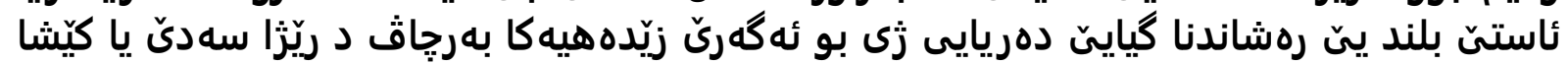

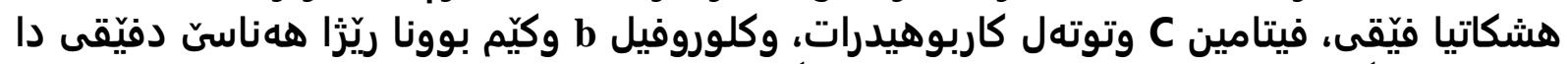

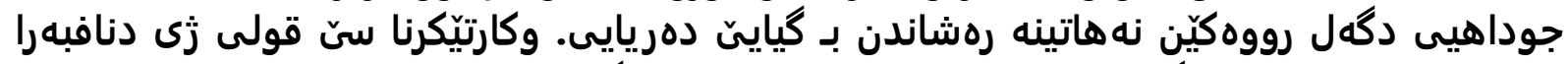

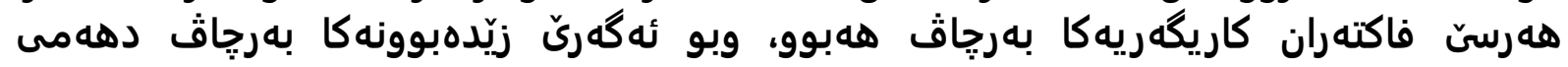

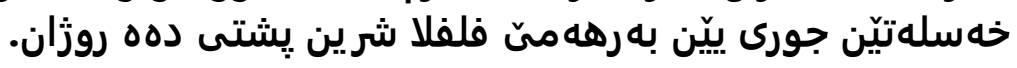

تأثير سماد الكومبوست ومستخلص الأعشاب البحرية على الصفات الفسلجية التسويقية لصنفين هجينين من الفلفل الحلو (Capsicum annuum L.)

اجريت هذه الخلاصة التجربة في حقول الخضروات، العائدة لكلية علوم الهندسة الزراعية جامعة

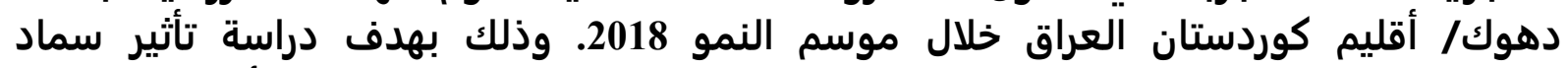

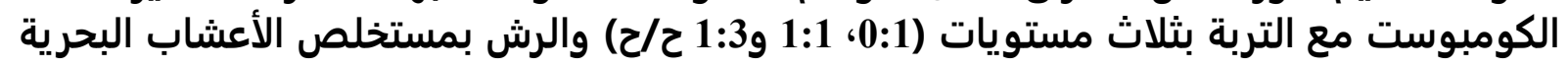




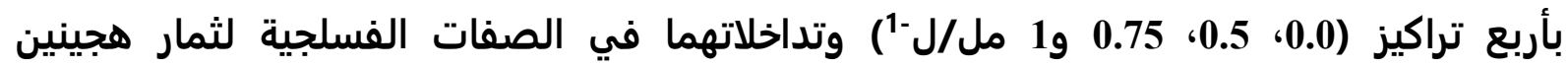

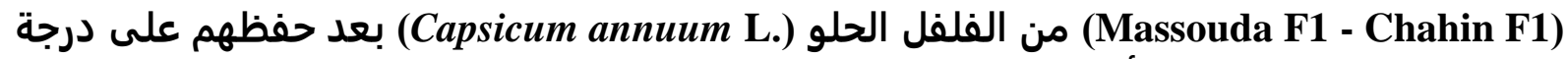

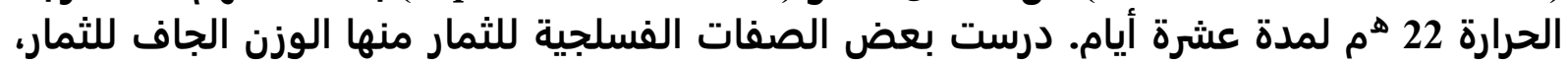

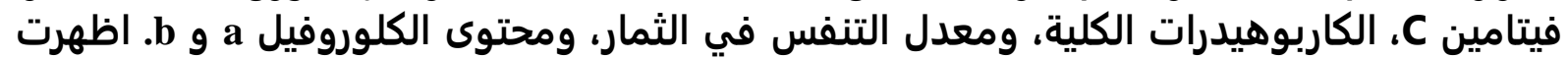

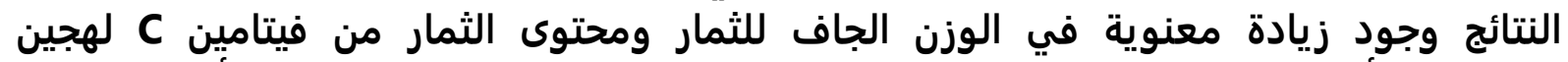

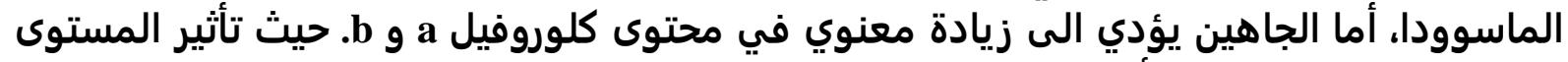

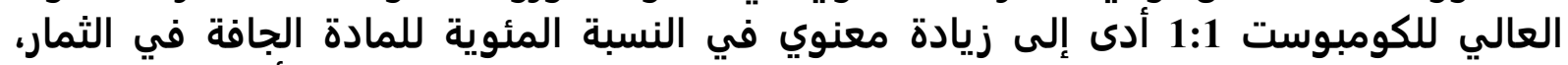

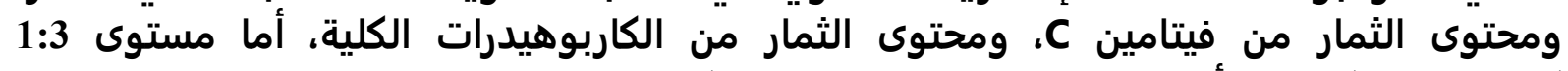

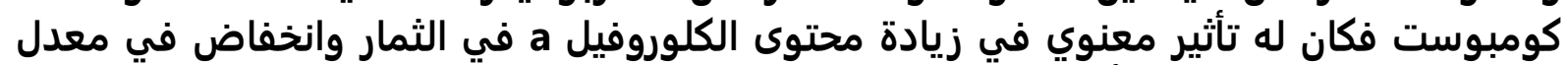

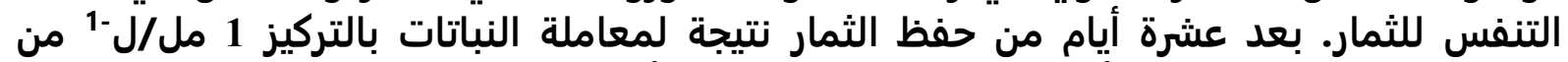

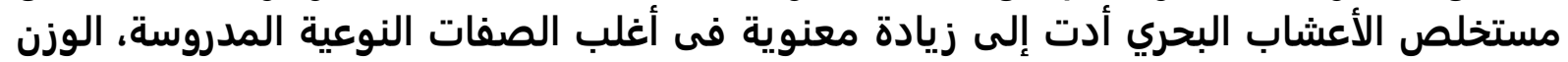

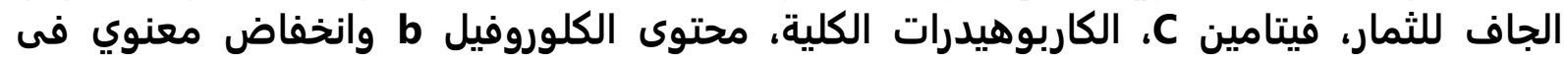

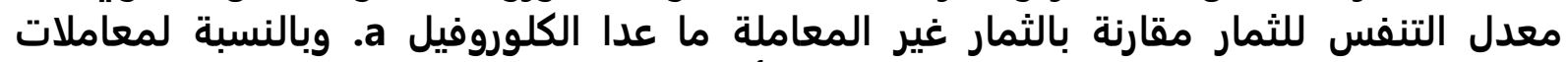
التداخل الثلاثي بين العوامل الثلاثة فقد سجلت الثمار أعلى المعدلات مقارلة مارنة بغير المعاملة ولجميع الصفات المدروسة. 